\title{
Chronic traumatic encephalopathy in an Iraqi war veteran with posttraumatic stress disorder who committed suicide
}

\author{
Bennet Omalu, M.D., M.B.A., M.P.H., C.P.E., ${ }^{1,2}$ Jennifer L. Hammers, D.O., ${ }^{1,3}$ \\ Julian Bailes, M.D. ${ }^{1,4}$ Ronald L. Hamilton, M.D., ${ }^{5}$ M. Ilyas Kamboh, Ph.D., ${ }^{6}$ \\ Garrett Webster, ${ }^{1,2}$ and Robert P. Fitzsimmons, J.D. ${ }^{1,7}$
}

\begin{abstract}
${ }^{1}$ Brain Injury Research Institute, Morgantown, West Virginia; ${ }^{2}$ Department of Pathology, University of California, Davis, California; ${ }^{3}$ Office of the Chief Medical Examiner, Boston, Massachusetts; ${ }^{4}$ Department of Neurosurgery, West Virginia University, Morgantown, West Virginia; ${ }^{5}$ Department of Pathology, University of Pittsburgh, Pennsylvania; ${ }^{6}$ Department of Human Genetics, University of Pittsburgh, Pennsylvania; and ${ }^{7}$ Fitzsimmons Law Offices, Wheeling, West Virginia
\end{abstract}

\begin{abstract}
Following his discovery of chronic traumatic encephalopathy (CTE) in football players in 2002, Dr. Bennet Omalu hypothesized that posttraumatic stress disorder (PTSD) in military veterans may belong to the CTE spectrum of diseases. The CTE surveillance at the Brain Injury Research Institute was therefore expanded to include deceased military veterans diagnosed with PTSD. The authors report the case of a 27 -year-old United States Marine Corps (USMC) Iraqi war veteran, an amphibious assault vehicle crewman, who committed suicide by hanging after two deployments to Fallujah and Ramadi. He experienced combat and was exposed to mortar blasts and improvised explosive device blasts less than $50 \mathrm{~m}$ away. Following his second deployment he developed a progressive history of cognitive impairment, impaired memory, behavioral and mood disorders, and alcohol abuse. Neuropsychiatric assessment revealed a diagnosis of PTSD with hyperarousal (irritability and insomnia) and numbing. He committed suicide approximately 8 months after his honorable discharge from the USMC. His brain at autopsy appeared grossly unremarkable except for congestive brain swelling. There was no atrophy or remote focal traumatic brain injury such as contusional necrosis or hemorrhage. Histochemical and immunohistochemical brain tissue analysis revealed CTE changes comprising multifocal, neocortical, and subcortical neurofibrillary tangles and neuritic threads (ranging from none, to sparse, to frequent) with the skip phenomenon, accentuated in the depths of sulci and in the frontal cortex. The subcortical white matter showed mild rarefaction, sparse perivascular and neuropil infiltration by histiocytes, and mild fibrillary astrogliosis. Apolipoprotein E genotype was 3/4. The authors report this case as a sentinel case of CTE in an Iraqi war veteran diagnosed with PTSD to possibly stimulate new lines of thought and research in the possible pathoetiology and pathogenesis of PTSD in military veterans as part of the CTE spectrum of diseases, and as chronic sequelae and outcomes of repetitive traumatic brain injuries. (DOI: 10.3171/2011.9.FOCUS11178)
\end{abstract}

\section{KEY WoRDS • posttraumatic stress disorder • Iraq • chronic traumatic encephalopathy $\quad$ - suicide}

$\mathrm{I}$ N 2002 Dr. Bennet Omalu ${ }^{22}$ discovered and described CTE in a football player when he performed an autopsy on Mike Webster. Since 2002, Dr. Omalu, the Brain Injury Research Institute, and other researchers have identified and described CTE in numerous football players, wrestlers, boxers, and ice hockey players, which have been reported in the literature. ${ }^{16,17,19-22,24,25}$ Following our elucidation of CTE in athletes, we hypothesized that PTSD in war veterans may belong to the CTE spectrum given that active military personnel are high-risk cohorts for repeated subconcussive and concussive trau-

\footnotetext{
Abbreviations used in this paper: $\mathrm{CTE}=$ chronic traumatic encephalopathy; DSM-IV-TR = Diagnostic and Statistical Manual of Mental Disorders, Fourth Edition, Text Revision; IED = improvised explosive device; PTSD = posttraumatic stress disorder; TDP-43 = TAR-DNA-binding protein 43; USMC = United States Marine Corps.
}

matic brain injuries; for example, bomb blasts can cause traumatic brain injuries from primary pressure wave and acceleration-deceleration injury mechanisms. ${ }^{4,28} \mathrm{We}$ expanded our CTE surveillance and brain tissue analyses to include deceased military veterans who were diagnosed with PTSD.

In 2010 we encountered CTE changes in the brain of a 61-year-old deceased Vietnam war veteran, who died suddenly as a result of coronary atherosclerotic disease. This case was reported in the Stars and Stripes news magazine of the Department of Defense. ${ }^{26}$ The case was not published because we did not have comprehensive access to the medical records and family and social histories. Approximately 1 year later we have identified CTE changes in the brain of a 27-year-old Iraqi war veteran who was diagnosed with PTSD and committed suicide by hanging.

In our 2010 CTE paper, ${ }^{20}$ we had defined CTE as 


\section{B. Omalu et al.}

a progressive neurodegenerative syndrome caused by single, episodic, or repetitive blunt force impacts to the head and transfer of acceleration-deceleration forces to the brain. Chronic traumatic encephalopathy presents clinically after a prolonged latent period as a composite syndrome of mood disorders and neuropsychiatric and cognitive impairment. Direct brain tissue analysis reveals multifocal or diffuse tauopathy, which may be accompanied by low-grade and multifocal white matter rarefaction, microglial activation, and parenchymal histiocytes. Amyloidopathy may be present; however, the primary proteinopathy in CTE is a tauopathy. Some patients with CTE may not exhibit the classic prolonged latency period before clinical symptoms begin.

Posttraumatic stress disorder in war veterans was first designated in 1978 to describe a condition in Vietnam war veterans, and the syndrome was first recognized by the American Psychiatric Association in the early 1980s..$^{30}$ A primary neurodegenerative proteinopathy has not been defined for PTSD in war veterans. Pathognomonic tissue neuropathological features have not been specified. Clinical diagnoses are currently based on presenting clinical symptomatology, based on two diagnostic systems, which continue to evolve as our understanding of PTSD continues to grow. ${ }^{30,32}$ Table 1 shows the DSMIV-TR clinical criteria for PTSD diagnosis, ${ }^{1}$ and Table 2 shows the ICD-10 clinical criteria for PTSD diagnosis. ${ }^{31}$

We report this case as a sentinel case of CTE in an Iraqi war veteran diagnosed with PTSD to possibly stimulate new lines of thought and research in the possible pathoetiology and pathogenesis of PTSD in military veterans as it relates to PTSD being part of the CTE spectrum of diseases, and as chronic sequelae and outcomes of repetitive traumatic brain injuries.

\section{Case Report}

\section{Premortem History}

This subject was a 27-year-old Caucasian man who committed suicide by hanging approximately 8 months after his honorable discharge from the USMC and while he was beginning a divorce process with his wife. His wife for 3 years, a 6-year-old stepson, and a 2-year-old biological son had left him and moved in with her parents. Reportedly, he had performed well in high school, obtaining mostly "A" grades. In college he began binge drinking and barely received passing grades, which he attributed to his binge drinking; however, he received a bachelor's degree in history. Following college he joined the USMC at the age of 23 and was honorably discharged after 4 years (2006-2010) with a rank of corporal. Before entering the military he had worked as a waiter and as a staff member in a national vitamin retail chain.

His listed military occupational specialty was 1833 Amphibious Assault Vehicle Crewman. He served two deployments to Iraq. The first deployment to Iraq was in 2007 for approximately 8 months in Fallujah, where he was assigned to mechanized mobile patrols. The second deployment occurred in 2008 for approximately 5 months in Ramadi, where he was assigned to an entry control point. He experienced combat and reported exposures to mortar blasts and IED blasts less than $50 \mathrm{~m}$ away. During the second deployment he was court marshaled twice for acting out, insubordination, fighting, hazing, and assault, and was dropped 1 rank. He described only a few incidents during his deployment that he found bothersome. There was an incident during the 3 rd week of his first deployment when he witnessed a vehicle in his patrol blown up, and marines killed and wounded. In another incident, approximately 2 weeks later, while hooking up their disabled vehicle to tow, 2 marines in his section were shot and he helped to patch them up. In yet another incident, he witnessed a school bus full of Iraqi citizens, many of whom were children, blown up by an IED.

After his deployments he was stationed at a base and played football in a base league. During a football game in 2009, approximately 9 months after his second deployment, he reported being hit from the side causing him to fall to the ground. He stood up, stumbled, fell again, and then continued the game. Other players noticed that he was confused and kept asking the count and details of the next play and he had to be removed from the game. The events of the following week were unclear and he reported residual headaches and memory problems. Reportedly a conventional CT scan of the head was performed and showed no significant findings. He reported playing football and hockey for leisure and was never diagnosed with a concussion, although he suffered his "bell rung."

Two days prior to a 2010 neuropsychological evaluation, he was involved in a single motor vehicle crash while he was driving under the influence of alcohol, when he turned a corner and flipped his car. He woke up later hanging upside down in the car. It was not clear whether he lost consciousness from a head injury or from stuporous alcohol intoxication. He noted the following morning that he suffered from headaches and vomited; however, it was not also clear whether these symptoms were alcoholrelated or head injury-related. He lost his driver's license after this crash for driving under the influence and refusing a blood-alcohol test. He visited a Veteran Affairs Medical Center the day before he committed suicide and reported having a new job as a football coach with his old high school, and was currently attending a community college. His driving under the influence charges had been dismissed.

In 2010, he was referred for a neuropsychological screening. His wife reported that he forgot dates, conversations, and trivialities of daily living. He also forgot whether he completed tasks, and sometimes confused his wife's and sister's names. He had problems making decisions and therefore avoided them. He believed he snapped at his children too frequently and was increasingly becoming a grumpy person. He admitted to headaches that occurred 3 to 4 times per week, which he described as pressure in his entire head. The headaches were relieved by a nonsteroidal antiinflammatory agent. He experienced bilateral hearing problems and tinnitus, which he dated back to when he had worked on engines in the military. He reported dizziness when he woke up at night to use the bathroom, slept only 4 hours a night, and had trouble falling asleep. Other reported symptoms included irrita- 


\section{Chronic traumatic encephalopathy in an Iraqi war veteran}

\section{TABLE 1: Diagnostic and Statistical Manual of Mental Disorders (DSM-IV-TR), Fourth Edition: Diagnostic Criteria for Posttraumatic Stress}

Disorder*

A. The person has been exposed to a traumatic event in which both of the following were present:

1. The person experienced, witnessed, or was confronted with an event or events that involved actual or threatened death or serious injury, or a threat to the physical integrity of self or others

2. The person's response involved intense fear, helplessness, or horror. Note: In children, this may be expressed instead by disorganized or agitated behavior

B. The traumatic event is persistently reexperienced in 1 [or more] of the following ways:

1. Recurrent and intrusive distressing recollections of the event including images, thoughts, or perceptions. Note: In young children, repetitive play may occur in which themes or aspects of the trauma are exposed.

2. Recurrent distressing dreams of the event. Note: In children, there may be frightening dreams without recognizable content.

3. Acting or feeling as if the traumatic event were recurring [includes a sense of reliving the experience, illusions, hallucinations, and dissociative flashback episodes, including those that occur on awakening or when intoxicated]. Note: In young children, trauma-specific reenactment may occur.

4. Intense psychological distress at exposure to internal or external cues that symbolize or resemble an aspect of the traumatic event

5. Physiological reactivity on exposure to internal or external cues that symbolize or resemble an aspect of the traumatic event

C. Persistent avoidance of stimuli associated with the trauma and numbing of general responsiveness (not present before the trauma), as indicated by 3 (or more) of the following:

1. Efforts to avoid thoughts, feelings, or conversations associated with the trauma

2. Efforts to avoid activities, places, or people that arouse recollections of the trauma

3. Inability to recall an important aspect of the trauma

4. Markedly diminished interest or participation in significant activities

5. Feeling of detachment or estrangement from others

6. Restricted range of affect (e.g., unable to have loving feelings)

7. Sense of foreshortened future (e.g., does not expect to have a career, marriage, children, or a normal life span)

D. Persistent symptoms of increased arousal (not present before the trauma), as indicated by 2 (or more) of the following:

1. Difficulty falling or staying asleep

2. Irritability or outbursts of anger

3. Difficulty concentrating

4. Hypervigilance

5. Exaggerated startle response

E. Duration of the disturbance (symptoms in Criteria B, C, and D) is more than 1 month.

F. The disturbance causes clinically significant distress or impairment in social, occupational, or other important areas of functioning.

Specify if:

Acute: if duration of symptoms is less than 3 months

Chronic: if duration of symptoms is 3 months or more

Specify if:

With Delayed Onset: if onset of symptoms is at least 6 months after the stressor

* Reprinted with permission from the DSM-IV-TR (Copyright @ 2000). American Psychiatric Association.

bility, discomfort with crowds, startle reactions, anhedonia, withdrawal and lack of engagement with the family, emotional numbing, and detachment. Many of his symptoms began after his first and second deployments. He also reported getting angry quickly and losing his temper frequently. He smoked cigarettes and reported drinking rarely; however, when he did drink, he drank a lot. When he got out of the marines he drank weekly. There was minor experimentation with marijuana and other drugs while he was in college. He was not aware of any family history of mental illness or any developmental impairment. His other significant medical history was a nasal bone fracture from an unspecified cause when he was 21 years old.

\section{Neuropsychological Testing}

The following neuropsychological tests and procedures were performed: Wechsler Test of Adult Intelligence; California Verbal Learning Test; Rey-Osterrieth Complex Figure Test; Grooved Pegboard Test; Finger Tapping Test; Trail Making Test; Word-list generation tasks; Ruff Figural Fluency Test; Ruff 2\&7 Selective Attention Test; Test of Memory Malingering; Beck Depression Inventory-2; and PTSD checklist-military version.

For general cognitive functioning, his baseline intellectual abilities were estimated to fall in the high average to superior range based on demographic variables as well as a word-reading test (Wechsler Test of Adult Intel- 
B. Omalu et al.

TABLE 2: World Health Organization ICD-10 classification of mental and behavioral disorders. Clinical descriptions and diagnostic guidelines*

\begin{abstract}
PTSD
This arises as a delayed and/or protracted response to a stressful event or situation (either short- or long-lasting) of an exceptionally threatening or catastrophic nature, which is likely to cause pervasive distress in almost anyone (e.g., natural or man-made disaster, combat, serious accident, witnessing the violent death of others, or being the victim of torture, terrorism, rape, or other crime). Predisposing factors such as personality traits (e.g. compulsive, asthenic) or previous history of neurotic illness may lower the threshold for the development of the syndrome or aggravate its course, but they are neither necessary nor sufficient to explain its occurrence.

Typical symptoms include episodes of repeated reliving of the trauma in intrusive memories ("flashbacks") or dreams, occurring against the persisting background of a sense of "numbness" and emotional blunting, detachment from other people, unresponsiveness to surroundings, anhedonia, and avoidance of activities and situations reminiscent of the trauma. Commonly there is fear and avoidance of cues that remind the sufferer of the original trauma. Rarely, there may be dramatic, acute bursts of fear, panic or aggression, triggered by stimuli arousing a sudden recollection and/or reenactment of the trauma or of the original reaction to it.

There is usually a state of autonomic hyperarousal with hypervigilance, an enhanced startle reaction, and insomnia. Anxiety and depression are commonly associated with the above symptoms and signs, and suicidal ideation is not infrequent. Excessive use of alcohol or drugs may be a complicating factor. The onset follows the trauma with a latency period, which may range from a few weeks to months (but rarely exceeds 6 months). The course is fluctuating but recovery can be expected in the majority of cases. In a small proportion of patients the condition may show a chronic course over many years and a transition to an enduring personality change.

Diagnostic Guidelines

This disorder should not generally be diagnosed unless there is evidence that it arose within 6 months of a traumatic event of exceptional severity. A "probable" diagnosis might still be possible if the delay between the event and the onset was longer than 6 months, provided that the clinical manifestations are typical and no alternative identification of the disorder (e.g., as an anxiety or obsessive-compulsive disorder or depressive episode) is plausible. In addition to evidence of trauma, there must be a repetitive, intrusive recollection or re-enactment of the event in memories, daytime imagery, or dreams. Conspicuous emotional detachment, numbing of feeling, and avoidance of stimuli that might arouse recollection of the trauma are often present but are not essential for the diagnosis. The autonomic disturbances, mood disorder, and behavioural abnormalities all contribute to the diagnosis but are not of prime importance.
\end{abstract}

* Reprinted with permission from the WHO.

ligence: estimated full-scale intelligence quotient $=119$, 90th percentile). For attention, he was able to attend to instructions throughout the evaluation. He occasionally required some limited repetition of directions. A measure of selective attention was in the average range for speed (58th percentile) and accuracy (62nd percentile). His performance improved slightly as the test progressed, which suggested intact sustained attention to the task. For cognitive/motor processing speed, connection of sequentially numbered circles was performed in the average range ( 25 seconds, 42nd percentile). Fine motor dexterity was in the average range bilaterally. The dominant-hand performance was 1 second slower than the nondominant hand (right 61 seconds, 42nd percentile; left 60 seconds, 69th percentile).

For executive functioning, a task that required the ability to establish and maintain an alternating alphanumeric sequence was performed in the average range (49 seconds, 58th percentile) with no errors. Word-list generation by letter was in the superior range (FAS $=17 / 18 / 25$, 92nd percentile), while generation by category was highaverage (28 animals, 76 th percentile). Design fluency was in the low-average range (14th percentile), with a few repetition errors (5 total, $42 \mathrm{nd}$ percentile). Approach on the first trial was efficient and productive, but his efficiency declined on the subsequent 4 trials, resulting in a lower total output. Copy of a complex design was impaired (less than first percentile), due largely to a highly segmented approach with no attention to the gestalt of the figure.

For learning and memory, his ability to learn a list of 16 words over 5 consecutive learning trials was in the average range (California Verbal Learning Test-2, 42nd percentile). His performance on a subsequent interference list was in the superior range (93rd percentile), reflecting a benefit from prior exposure to analogous task. Free recall following this interference list was in the average range (12 words, 69 th percentile). Cuing did not change his performance (50th percentile). After a longer delay, his recall was at the 31 st percentile, and with cuing, at the 14th percentile. He committed 11 intrusion errors across trials (second percentile): about half were the same single word, which he listed on each learning trial and 1 recall trial, and the remainder represented source memory errors. Yes/no recognition revealed a positive response bias (15/16 hits, 50th percentile; 6 false positive errors, most of which were source-recognition errors and all of which were semantically related, 7th percentile). Forced-choice recognition was errorless (16/16). Incidental memory for a complex design was in the impaired range for both immediate (second percentile) and delayed (first percentile) conditions, likely due in large part to his aforementioned fractured approach to the initial copy of the figure.

\section{Diagnosis of PTSD}

His final diagnoses were the following: Axis 1: PTSD with hyperarousal (irritability and insomnia) and numbing, alcohol abuse, continuous; Axis 2: deferred; Axis 3: hyperlipidemia; Axis 4: combat deployment, reintegration difficulties, unemployment, recent arrest, marital stress. His prescribed neurotropic medications included 


\section{Chronic traumatic encephalopathy in an Iraqi war veteran}

citalopram, trazodone, and prazosin. In a clinic visit 2 months prior to his suicide, he reported persistent PTSD symptoms.

\section{Postmortem History}

The subject's parents had requested the police perform a well-being check of their son after they had not heard from him for 2 days. The police found our subject in his residence hanging from a staircase by a leather belt noose around his neck. A full forensic autopsy by the combined technique of Virchow and Rokitansky ${ }^{14}$ was performed by the medical examiner. After informed consent was granted by the wife and next-of-kin, his brain was forwarded to the Brain Injury Research Institute for gross neuropathological, histochemical, and immunohistochemical analysis.

\section{Autopsy Findings}

At autopsy our subject was unclad, appeared welldeveloped and well-nourished, weighed approximately 211 pounds, measured approximately 74 inches, and appeared consistent with the stated age of 27 years old. There was a ligature, which was tightly wound around the neck in a noose, and composed of a brown braided leather belt, which was looped through a buckle located on the posterior neck. The underlying ligature indentation mark was situated circumferentially around the neck in a transverseoblique-ascending fashion. There were no anterior strap muscle hemorrhages or fractures of the hyoid bone or thyroid cartilage.

Dissection of the thoracic and abdominal cavities revealed normally situated viscera, which were grossly and histologically unremarkable. The scalp revealed no contusional hemorrhages. Dissection of the cranial cavity revealed no skull fractures and no intracranial hemorrhages. Toxicological analysis of the fluoridated heart blood and vitreous humor revealed the presence of citalopram only without any other drug detected. A sample of whole heart blood was submitted for apolipoprotein E genotyping in an ethylenediaminetetraacetic acid specimen bottle. The underlying cause of death was determined to be asphyxiation due to hanging and the manner of death was determined to be a suicide.

\section{Gross Neuropathological Findings}

The brain was fixed in $10 \%$ buffered formaldehyde for 2 weeks before it was grossed. The dura mater revealed no xanthochromia, membranes, or hemorrhages. The weight of the formaldehyde fixed brain was 1624 grams. The cerebral and cerebellar hemispheres appeared symmetrical and exhibited gyral, sulcal, and folial convolutions that appeared normal. There was diffuse global and symmetrical expansion of gyri and compression of sulci, accompanied by symmetrical bilateral grooving of the unci and cerebellar tonsils, with symmetrical compression of the subarachnoidal cisterns. The arachnoid and pia mater appeared smooth and glistening without acute or chronic subarachnoidal hemorrhages. There was no acute or chronic cerebral cortical contusional or ischemic necrosis or hemorrhage. There was no lobar cortical atrophy.
The centrum semiovale and the periventricular white matter revealed very focal decompositional change and Swiss-cheese appearance, accompanied by central redpink parenchymal discoloration, edema, and congestion. There was no periventricular leukomalacia, demyelinating plaques, necrosis, hemorrhage, or infarct in the subcortical white matter. There was no acute or chronic gliding contusional hemorrhage or necrosis.

The ventricles were symmetrically compressed and showed no acute or chronic intraventricular hemorrhage. The genu, body, and splenium of the corpus callosum revealed no hemorrhages or necrosis. All subcortical nuclei, including the caudate nucleus, putamen, globus pallidus, thalamus, and subthalamic nucleus, on both sides, revealed no atrophy, necrosis, or hemorrhage (Fig. 1 upper). The hippocampus and parahippocampal gyrus, on both sides, were not dysplastic or atrophic (Fig. 1 lower).

The midbrain, pons, and medulla oblongata revealed no atrophy, dorsolateral hemorrhage, or collicular and teg-

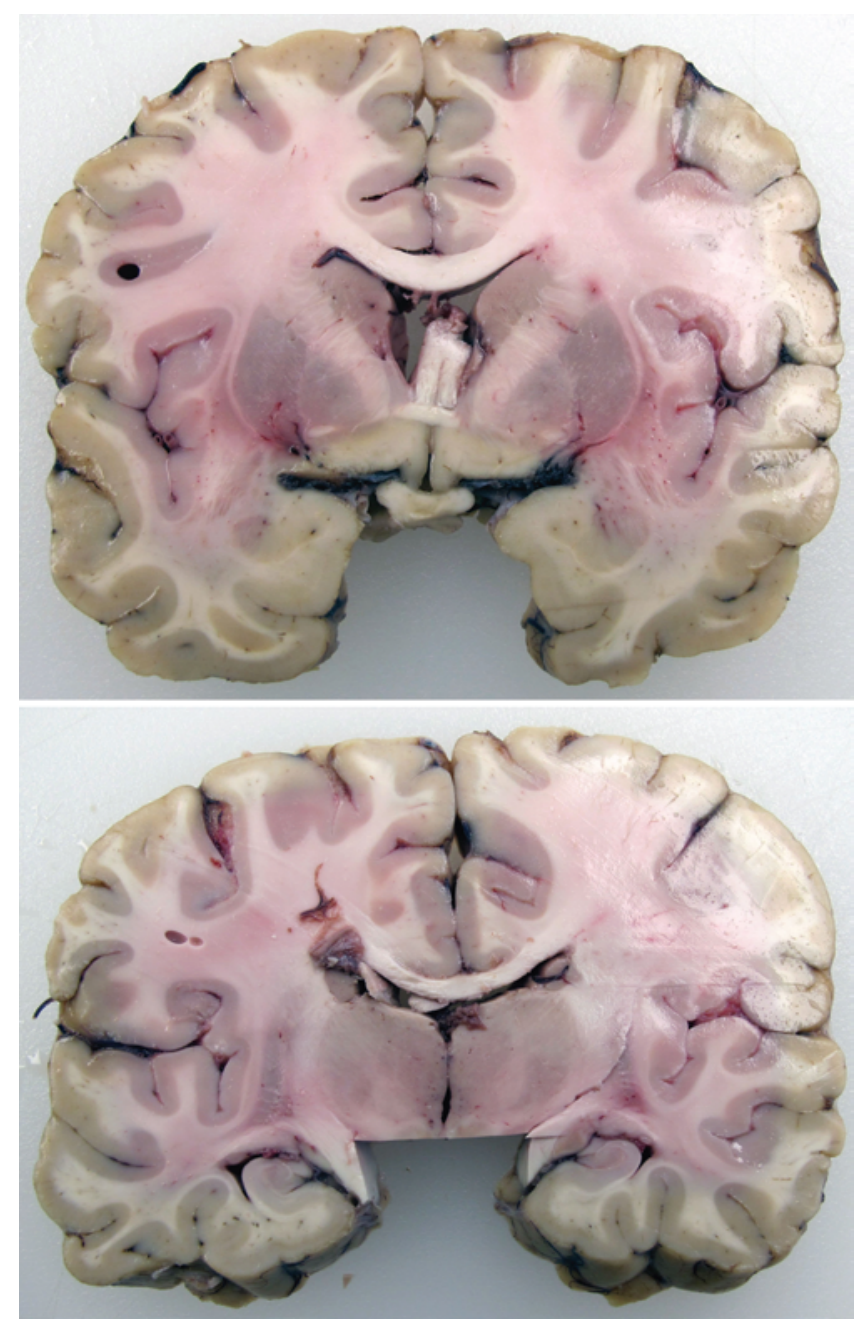

Fig. 1. Gross photographs of the coronal section of the brain at the level of the anterior commissure (upper) and hippocampus (lower), showing diffuse parenchymal edema with very focal Swiss-cheese change, without atrophy of the subcortical ganglia (upper), hippocampus or thalamus (lower), or any other focal gross parenchymal gray or white matter lesions. 


\section{B. Omalu et al.}

mental necrosis or hemorrhage. The basis pontis showed no central myelinolysis or Duret hemorrhage. The cerebral peduncles, medullary pyramids, and cerebellar peduncles were not atrophic and showed no hemorrhage or necrosis. The substantia nigra and locus ceruleus were adequately pigmented for age. The inferior olivary nuclei and dentate nuclei revealed normal convolutional patterns. The cerebellar hemispheres revealed no folial atrophy or necrosis. There were no hemorrhages or infarcts in the cerebellar cortex or white matter. The pituitary gland appeared unremarkable. The spinal cord revealed no epidural, subdural, or subarachnoidal hemorrhage. The spinal medulla appeared unremarkable without segmental atrophy, contusional necrosis or hemorrhage, or white matter funicular degeneration or demyelination.

Thirty-one representative topographically selected sections of the dura mater, neocortex, subcortical ganglia, brainstem, pituitary gland, and spinal cord were obtained and stained by the following histochemical and immunohistochemical tissue staining protocols: 1) H \& E; 2) tau; 3) $\beta$-A4 amyloid; 4) $\alpha$-synuclein; 5) glial fibrillary acidic protein; 6) CD-68; 7) Luxol fast blue and cresyl violet; 8) ubiquitin; 9) TDP-43; and 10) Bielschowsky silver impregnation stain.

\section{Microscopic Neuropathological Findings}

$H \& E$ Stains. The frontal, parietal, temporal, occipital, insular, and cingulate cortex revealed the expected columnar and laminar organization without cortical disorganization or dysplasia. There was negligible-to-mild neuronal dropout without eosinophilic neuronal necrosis. There was diffuse perineuronal vacuolation, expansion of Virchow Robin spaces and patchy neuropil microspongiosis of both the gray and white matter. There was marked congestion of the arachnoid and pia mater and the penetrating parenchymal vessels. Multifocal sparse perivascular pigment-laden histiocytes were noted in many Virchow Robin spaces.

The body and splenium of the corpus callosum, internal, external, and extreme capsules revealed no necrosis, hemorrhage, rarefaction, myelinolysis, or axonolysis. The periventricular white matter revealed no leukomalacia or subependymal astrogliosis. The subcortical ganglia including the caudate nucleus, putamen, globus pallidus, hypothalamus, thalamus, amygdala, and basal nucleus of Meynert showed only diffuse neuropil extracellular edema. There was no mineralization of the walls of the vessels of the globus pallidus or cornu ammonis.

The dentate gyrus, cornu ammonis, subiculum, and entorhinal cortex of the hippocampus revealed no significant neuronal dropout, dysplasia, or sclerosis. There was no eosinophilic necrosis of the pyramidal neurons of the stratum pyramidalis. The midbrain, pons, and medulla revealed parenchymal edema without any other focal or diffuse lesions. The neurons of the substantia nigra, locus ceruleus, and dorsal raphe nucleus were adequately pigmented and revealed no neuronal dropout, Marinesco bodies, pale bodies, or Lewy bodies. The cerebellar cortex revealed negligible Purkinje neuron dropout and Bergmann astrogliosis. The internal granule cell layer and dentate nucleus were unremarkable. The cerebellar white matter revealed no rarefaction, necrosis, or infarcts. The dura mater revealed marked intradural congestion without inflammation, or acute or remote hemorrhage. The adenohypophysis revealed focal interstitial fibrosis and marked sinusoidal congestion. The neurohypophysis was congested and edematous. The spinal medulla revealed the normal gray and white matter morphology without significant loss of the anterior horn neurons, which showed sparse nonspecific cytoplasmic neuronal changes. The white matter funiculi and tracts were unremarkable.

Other Histochemical and Immunohistochemical Stains. Tau-immunostained sections revealed none, to sparse, to multifocally frequent band- and flame-shaped neurofibrillary tangles and neuritic threads in the frontal cortex, parietal cortex, temporal cortex, occipital cortex, and cingulate cortex with a skip phenomenon, ${ }^{20}$ accentuated in the depths of sulci (Fig. 2). The largest numbers of tangles and threads were noted in the frontal cortex, with none in the occipital cortex. The CA1 region and subiculum revealed none to sparse flame, band, and small globose neurofibrillary tangles and neuritic threads. The entorhinal cortex revealed moderate band- and flameshaped neurofibrillary tangles and neuritic threads. There was focally sparse to moderate flame- and band-shaped neurofibrillary tangles in the amygdala. The CA2, CA3, and CA4 regions of the cornu ammonis showed no neurofibrillary tangles in the pyramidal neurons and no neuritic threads. The granule neurons of the dentate fascia revealed no neurofibrillary tangles or neuritic threads. There was focally sparse flame- and band-shaped neurofibrillary tangles and neuritic threads in the anterior perforated substance and basal nucleus of Meynert. The thalamus and hypothalamus showed several large globose neurofibrillary tangles and neuritic threads. The substantia nigra showed none to sparse small and large globose neurofibrillary tangles and neuritic threads. The caudate nucleus, insula cortex, putamen, and globus pallidus revealed no neurofibrillary tangles. Only 1 neuritic thread was noted in the globus pallidus. There were no neurofibrillary tangles in the midbrain tegmentum. Few pretangles were noted in neurons of the periaqueductal gray matter. The ventral tegmental pons revealed a single large globose neurofibrillary tangle and several neuritic threads. A single neuron revealed a globose neurofibrillary tangle in the locus ceruleus, accompanied by several neuritic threads. The medullary tegmentum and the inferior olivary nuclei revealed no neurofibrillary tangles or neuritic threads. The Purkinje neurons, internal granule neurons, and the dentate neurons of the cerebellum revealed no neurofibrillary tangles. The anterior horn neurons of the spinal cord revealed no neurofibrillary tangles. There were no neuritic threads in the spinal gray or white matter. Tufted astrocytes, thorn astrocytes, astrocytic plaques, and astrocytic coils and grains were absent in all sections of the brain examined.

There was focal ubiquitin immunopositivity of few neurofibrillary tangles and neuritic threads, otherwise there were no ubiquitin neuronal or glial inclusions in all sections of the brain examined. There were no diffuse or 


\section{Chronic traumatic encephalopathy in an Iraqi war veteran}

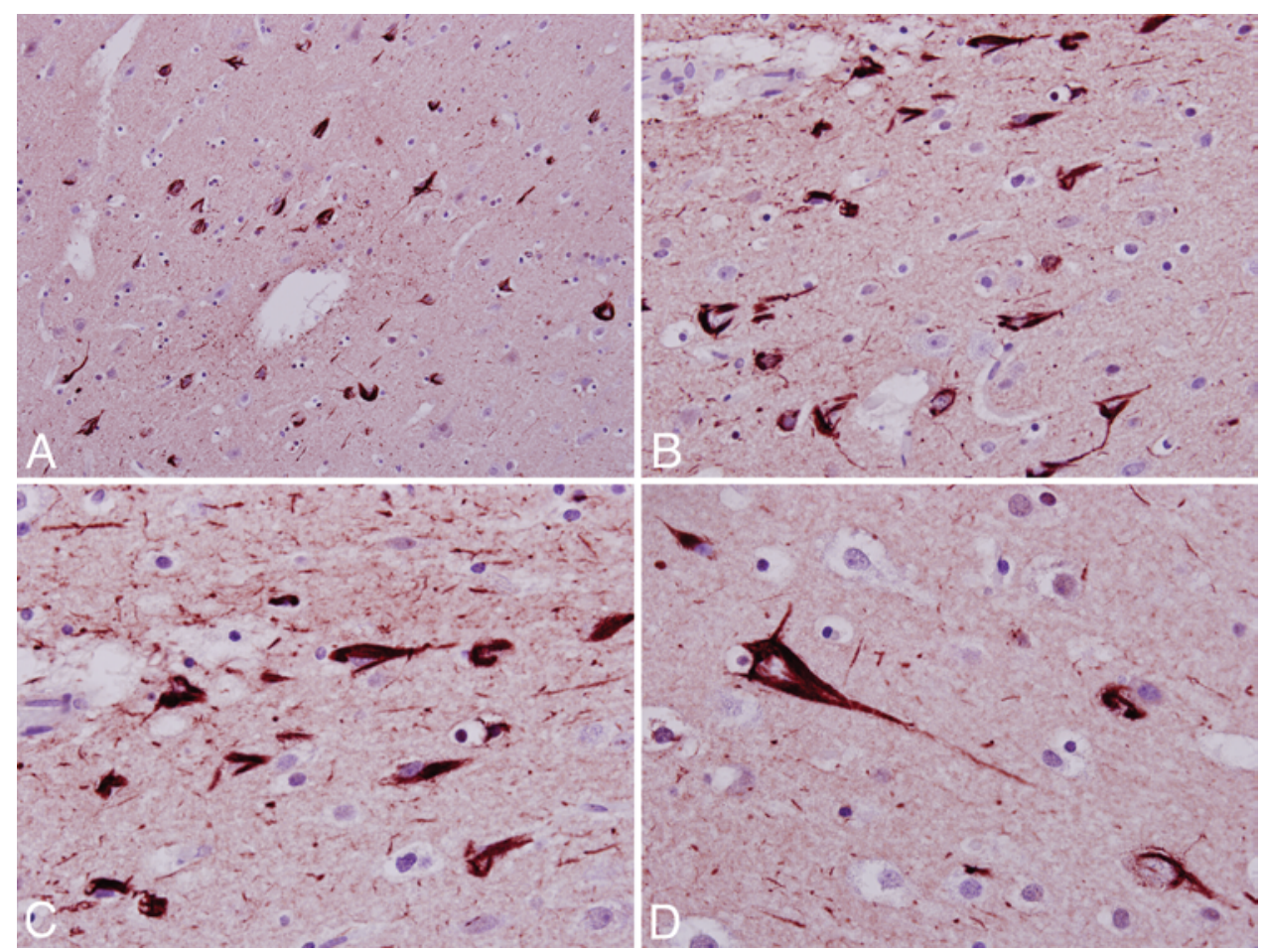

FIG. 2. Photomicrographs of tau-immunostained section of the frontal cortex showing frequent neurofibrillary tangles and neuritic threads $(A$ and $B)$, with higher magnification ( $C$ and $D$ ) showing band- and flame-shaped neurofibrillary tangles and neuropil neuritic threads. Original magnification $\times 200(A), \times 400(B), \times 600(C$ and D).

neuritic amyloid plaques and no cerebral amyloid angiopathy in all examined sections of the cortex, hippocampal formation, subcortical ganglia, brainstem, cerebellum, and spinal medulla. There were no Lewy bodies, Lewy neuritis, or glial alpha-synuclein inclusions in all examined sections of the cortex, hippocampal formation, subcortical ganglia, brainstem, cerebellum, and spinal medulla.

Glial fibrillary acidic protein immunostains revealed multifocal mild perivascular fibrillary astrogliosis and activation of astrocytes in the gray cortex and molecular layer of the frontal cortex, cingulate cortex, parietal cortex, temporal cortex, and occipital cortex. The subcortical gyral white matter of the cortical lobes revealed multifocal and superficial white matter fibrillary astrogliosis and activation of astrocytes. The body and splenium of the corpus callosum showed multifocal and mild fibrillary astrogliosis and activation of astrocytes. There was mild diffuse fibrillary astrogliosis of the hippocampus including the dentate fascia and gyrus, alveus, strata oriens, radiatum, lacunosum, and moleculare. There was mild diffuse astrogliosis of the thalamus, midbrain, pons, and medulla oblongata. There was diffuse astrogliosis of the neurohypophysis. The cerebellum revealed Bergmann astrogliosis with multifocal fibrillary astrogliosis of the superficial folial cerebellar white matter. There was mild diffuse fibrillary astrogliosis of the hypothalamus, amygdala, and anterior perforated substance (basal nucleus of Meynert). The anterior, lateral, and posterior cervical, thoracic, and lumbar funiculi did not show any remarkable astrogliosis.

The CD-68-immunostained sections of the frontal cortex, parietal cortex, temporal cortex, occipital cortex, cingulate gyrus, caudate nucleus, insula cortex, putamen, thalamus, hypothalamus, globus pallidus, cerebellum, corpus callosum, midbrain, pons, and medulla revealed nonspecific multifocal lysosomal astrocytic and microglial staining, accompanied by multifocal immunopositive staining of scattered perivascular histiocytes in and around the Virchow Robin spaces. Small clusters of neuropil histiocytes were present multifocally accentuated in the frontal and parietal subcortical white matter, internal capsule, anterior body of the corpus callosum, splenium of the corpus callosum, anterior commissure, cerebral peduncles, and transverse and longitudinal fascicles of the basis pontis. Similar changes were also noted in the cervical, thoracic, and lumbar anterior, lateral, and posterior funiculi, as well as in the amygdala and anterior perforated substance.

The sections stained with Luxol fast blue and cresyl violet from the frontal, temporal, parietal, and occipital cortex revealed mild diffuse rarefaction of the subcortical white matter, relatively sparing the occipital cortex, and accentuated in the frontal and parietal cortex. There was mild to moderate rarefaction of the subcortical white matter of the cingulate cortex, periventricular white matter of the lateral angle of the lateral ventricle, and of the anterior corpus callosum, as well as of the anterior commissure. There was diffuse mild rarefaction of the internal, external, and extreme capsules. Minimal rarefaction of the cerebral peduncle and medullary pyramids was noted. There was mild to moderate rarefaction of the transverse and longitudinal fasciculi of the basis pontis. There was no rarefaction of the deep cerebellar white matter. The splenium of the corpus callosum revealed mild rarefac- 


\section{B. Omalu et al.}

tion. There was no rarefaction or myelinolysis of the spinal anterior, lateral, or posterior funiculi.

The TDP-43-immunostained sections of the left frontal cortex, parietal cortex, cingulate cortex, insula cortex, claustrum, caudate nucleus, putamen, globus pallidus, and thalamus revealed very sparse and focal TDP-immunopositive cytoplasmic and neuritic inclusions in the frontal cortex and caudate nucleus. The pyramidal neurons of the cornu ammonis and the granule neurons of the dentate fascia revealed no TDP proteinopathy. The midbrain tegmentum, colliculi, periaqueductal gray matter, and substantia nigra showed very sparse and focal TDP nuclear and cytoplasmic inclusions in the substantia nigra. The Purkinje cell layer, internal granule cell layer, and dentate nucleus revealed no TDP proteinopathy or cytoplasmic inclusions. The anterior horn neurons of the spinal medulla revealed sparse and focal nuclear TDP inclusions accompanied by sparse dystrophic TDP-positive neurites.

The specified constellation of gross neuropathological, histomorphological, and immunophenotypical findings in this case are consistent with changes we have described and identified in CTE in American athletes. ${ }^{20}$

\section{Apolipoprotein E Genotyping}

Apolipoprotein E genotyping was performed using genomic DNA extracted from peripheral blood leukocytes in whole autopsy blood using the QIAamp DNA Blood Mini kit (Qiagen). The apolipoprotein E genotype was ascertained from the amplified DNA by fluorogenic $5^{\prime}$ nuclease assays (TaqMan SNP genotyping assays; Applied Biosystems). The apolipoprotein $\mathrm{E}$ genotype was 3/4.

\section{Discussion}

In this report we present the sentinel case of a 27-yearold Iraqi war veteran who was formally diagnosed with PTSD, committed suicide by hanging, and whose brain at autopsy revealed gross, histomorphological, and immunophenotypical findings that we have identified and described in CTE in American athletes. ${ }^{20}$ As in our previous CTE cases, ${ }^{19-25}$ the primary proteinopathy we identified was a tauopathy in the form of multifocal sparse to frequent topographic neurofibrillary tangles and neuritic threads with a skip phenomenon. ${ }^{20}$ Focal secondary TDP proteinopathy was identified. Nonspecific white matter changes accompanied the tauopathy, consisting of nonspecific fibrillary astrogliosis, white matter rarefaction, and perivascular and neuropil infiltration by histiocytes. There was no significant cortical, subcortical, or hippocampal cerebral atrophy. Acute or chronic focal traumatic brain injuries such as lobar contusional necrosis or hemorrhages were absent

Apolipoprotein E genotype in this case was 3/4. The $\mathrm{E} 3$ allele is the most recurrent apolipoprotein $\mathrm{E}$ allele we are observing in our CTE cohort, ${ }^{20}$ although our sample sizes are small and this observation is preliminary. We currently have no conclusive explanation for this trend that we are observing because the apolipoprotein E4 allele has been reported to be the high-risk allele for Alzheimer disease and adverse outcomes of traumatic brain injury. ${ }^{2,8}$ However, we suspect that E4 may not be the pri- mary apolipoprotein E risk allele for CTE although it has been suggested to be associated with chronic traumatic brain injury and cognitive impairment in boxers and professional football players. ${ }^{11,13,29}$ Kristman et al.. ${ }^{12}$ however, did not find any important association between the E4 allele and the risk of sustaining a concussion in amateur collegiate athletes.

Our subject was a member of a population cohort that can be exposed to repetitive traumatic brain injuries. Traumatic brain injury has been referred to as the signature injury of Operation Enduring Freedom and Operation Iraqi Freedom. It has been estimated that 15\%-30\% of troops engaged in active combat in Afghanistan and Iraq have suffered concussions and subconcussions, many as a result of blasts from explosive devices. The majority of these patients manifest neuropsychiatric symptoms and cognitive impairments beginning immediately after the injury and lasting for days to weeks to months. Approximately $18 \%-30 \%$ of these patients develop persistent, progressive, and sometimes disabling constellations of neuropsychiatric and cognitive impairments, which are interpreted to represent PTSD. ${ }^{3}$ Our subject also played football and hockey for leisure and suffered a remote nasal bone fracture. It is our belief that his eventual CTE risk outcome occurred as a result of his lifetime and cumulative exposure to repeated subconcussive and concussive traumatic brain injuries, with his military exposures being the primary injuries that precipitated CTE.

Our subject manifested persistent symptoms of CTE or PTSD and eventually committed suicide by hanging. We have previously associated parasuicides and suicides with $\mathrm{CTE}^{21}$ and are observing an overrepresentation of suicides and drug-related accidental deaths in our CTE cohort. ${ }^{20}$ According to a 2010 Department of Defense report ${ }^{6}$ the suicide rates in all services increased from about 10 per 100,000 people in 2001 to about 20 per 100,000 people in 2009, while the suicide rate in the general US population remained at approximately 11.5 per 100,000 in 2007. The propositional question that this sentinel case report raises is: What role does CTE play in the increasing incidence of suicides in the US military? This question can only be answered by more forensic observational and translational research focused on CTE and suicides in military veterans.

Members of the armed forces can sustain repetitive traumatic brain injury from training activities, from noncombat professional activities, and from combat activities. Blast exposure is the most common cause of traumatic brain injury in the wars in Iraq and Afghanistan. ${ }^{7}$ Explosives like mortar shells, rocket-propelled grenades, and IEDs cause blast injuries via complex physical events, which have the potential to precipitate repetitive subconcussive and concussive brain injuries. The mechanisms of brain damage are not yet well understood, but several neurotrauma mechanisms have been proposed. . $^{4,27}$

While emphasizing that no human autopsy studies, conducted with current immunohistochemical methods, have been published on blast-related traumatic brain injury in the military, Mac Donald et al., ${ }^{15}$ using diffusion tensor imaging, concluded that blast exposure in US military personnel causes traumatic axonal injury. Follow-up 


\section{Chronic traumatic encephalopathy in an Iraqi war veteran}

diffusion tensor imaging 6 to 12 months after preliminary postexposure diffusion tensor imaging confirmed persistence of traumatic axonal injury abnormalities in the brains of their military subjects. Their conclusions and findings support the conclusions, interpretations, and propositional sentinel value of this case report. The primary proteinopathy in CTE is hyperphosphorylated tau, which is a microtubule-associated protein that is involved in the pathophysiological cascades of traumatic axonal injury. ${ }^{10}$ We observed nonspecific white matter changes in this case, which accompanied tauopathy.

In addition to tauopathy in this case, we observed a focal and sparse secondary TDP proteinopathy. Such a TDP proteinopathy frequently occurs as a nondiagnostic or nondefining accompaniment and secondary proteinopathy of a variety of primary proteinopathies in a broad spectrum of neurodegenerative diseases, including Alzheimer disease..$^{5,9,18}$ Secondary proteinopathies in neurodegenerative diseases is a frequent occurrence, and CTE, as a trauma-induced neurodegenerative disease, may be accompanied by secondary proteinopathies in different cases of CTE.

\section{Conclusions}

In this paper we present the case of a 27-year-old USMC Iraqi war veteran who developed persistent impaired neuropsychiatric and cognitive functioning, and mood disorders, following deployments to Iraq and an honorable discharge. He was clinically diagnosed with PTSD and prescribed neurotropic drugs. He eventually committed suicide by hanging. Autopsy, as well as gross and histomorphological examination of his brain, revealed CTE changes similar to the CTE changes we have observed in American athletes. Chronic traumatic encephalopathy is the cumulative outcome of repeated subconcussive and concussive brain injuries, and in this instance, it is our opinion that the decedent sustained repeated subconcussive and concussive brain injuries primarily from exposures from blasts and secondarily from training activities and noncombat activities as a marine. Other possible tertiary nonmilitary contributory factors to his cumulative risk of developing CTE may have included a remote traumatic history of nasal bone fracture and engagement in contact sports such as football and hockey for leisure. This sentinel case highlights the need for forensic, observational, and translational research to further confirm that a proportion of PTSD cases in war veterans may be due to, or contributed to by, CTE caused by repeated subconcussive and concussive traumatic brain injuries.

\section{Disclosure}

The authors report no conflict of interest concerning the materials or methods used in this study or the findings specified in this paper.

Author contributions to the study and manuscript preparation include the following. Conception and design: Omalu, Hammers, Bailes, Fitzsimmons. Acquisition of data: Omalu, Hammers, Hamilton, Kamboh, Webster. Analysis and interpretation of data: Omalu, Hamilton, Kamboh. Drafting the article: Omalu. Critically revising the article: Omalu, Hammers, Bailes, Fitzsimmons.
Reviewed submitted version of manuscript: Omalu, Hammers, Bailes, Webster, Fitzsimmons. Approved the final version of the manuscript on behalf of all authors: Omalu. Administrative/technical/material support: all authors. Study supervision: Omalu, Bailes, Fitzsimmons.

\section{References}

1. American Psychiatric Association: Diagnostic and Statistical Manual of Mental Disorders, ed 4, Text Revision (DSM-IV-TR). Washington, DC: American Psychiatric Association, 2000

2. Bales KR, Dodart JC, DeMattos RB, Holtzman DM, Paul SM: Apolipoprotein E, amyloid, and Alzheimer disease. Mol Interv 2:339, 363-375, 2002

3. Belanger HG, Kretzmer T, Vanderploeg RD, French LM: Symptom complaints following combat-related traumatic brain injury: relationship to traumatic brain injury severity and posttraumatic stress disorder. J Int Neuropsychol Soc 16:194-199, 2010

4. Chen Y, Huang W: Non-impact, blast-induced mild TBI and PTSD: concepts and caveats. Brain Inj 25:641-650, 2011

5. Chen-Plotkin AS, Lee VM, Trojanowski JQ: TAR DNA-binding protein 43 in neurodegenerative disease. Nat Rev Neurol 6:211-220, 2010

6. Department of Defense Task Force on the Prevention of Suicide by Members of the Armed Forces: The Challenge and the Promise: Strengthening the Force, Preventing Suicide and Saving Lives. Washington, DC: US Department of Defense, 2010, p 233

7. Elder GA, Mitsis EM, Ahlers ST, Cristian A: Blast-induced mild traumatic brain injury. Psychiatr Clin North Am 33: 757-781, 2010

8. Ellison D, Love S, Chimelli L, Harding BN, Lowe J, Vinters HV: Neuropathology: A Reference Text of CNS Pathology, ed 2. New York: Mosby, 2004

9. Geser F, Lee VM, Trojanowski JQ: Amyotrophic lateral sclerosis and frontotemporal lobar degeneration: a spectrum of TDP-43 proteinopathies. Neuropathology 30:103-112, 2010

10. Greenfield JG, Love S, Louis DN, Ellison D: Greenfield's Neuropathology, ed 8. London: Hodder Arnold, 2008

11. Jordan BD, Relkin NR, Ravdin LD, Jacobs AR, Bennett A, Gandy S: Apolipoprotein E epsilon4 associated with chronic traumatic brain injury in boxing. JAMA 278:136-140, 1997

12. Kristman VL, Tator CH, Kreiger N, Richards D, Mainwaring L, Jaglal S, et al: Does the apolipoprotein epsilon 4 allele predispose varsity athletes to concussion? A prospective cohort study. Clin J Sport Med 18:322-328, 2008

13. Kutner KC, Erlanger DM, Tsai J, Jordan B, Relkin NR: Lower cognitive performance of older football players possessing apolipoprotein E epsilon4. Neurosurgery 47:651-658, 2000

14. Ludwig J: Handbook of Autopsy Practice, ed 3. Totowa, NJ: Humana Press, 2002

15. Mac Donald CL, Johnson AM, Cooper D, Nelson EC, Werner NJ, Shimony JS, et al: Detection of blast-related traumatic brain injury in U.S. military personnel. $\mathbf{N}$ Engl J Med 364:2091-2100, 2011

16. McKee AC, Cantu RC, Nowinski CJ, Hedley-Whyte ET, Gavett $\mathrm{BE}$, Budson $\mathrm{AE}$, et al: Chronic traumatic encephalopathy in athletes: progressive tauopathy after repetitive head injury. J Neuropathol Exp Neurol 68:709-735, 2009

17. McKee AC, Gavett BE, Stern RA, Nowinski CJ, Cantu RC, Kowall NW, et al: TDP-43 proteinopathy and motor neuron disease in chronic traumatic encephalopathy. J Neuropathol Exp Neurol 69:918-929, 2010

18. Neumann M, Sampathu DM, Kwong LK, Truax AC, Micsenyi $\mathrm{MC}$, Chou TT, et al: Ubiquitinated TDP-43 in frontotemporal lobar degeneration and amyotrophic lateral sclerosis. Science 314:130-133, 2006 


\section{B. Omalu et al.}

19. Omalu B: Play Hard, Die Young: Football Dementia, Depression, and Death. Lodi, CA: Neo-Forenxis Books, 2008

20. Omalu B, Bailes J, Hamilton RL, Kamboh MI, Hammers J, Case M, et al: Emerging histomorphologic phenotypes of chronic traumatic encephalopathy in American athletes. Neurosurgery 69:173-183, 2011

21. Omalu BI, Bailes J, Hammers JL, Fitzsimmons RP: Chronic traumatic encephalopathy, suicides and parasuicides in professional American athletes: the role of the forensic pathologist. Am J Forensic Med Pathol 31:130-132, 2010

22. Omalu BI, DeKosky ST, Hamilton RL, Minster RL, Kamboh MI, Shakir AM, et al: Chronic traumatic encephalopathy in a National Football League player: Part II. Neurosurgery 59: 1086-1093, 2006

23. Omalu BI, DeKosky ST, Minster RL, Kamboh MI, Hamilton RL, Wecht CH: Chronic traumatic encephalopathy in a National Football League player. Neurosurgery 57:128-134, 2005

24. Omalu BI, Fitzsimmons RP, Hammers J, Bailes J: Chronic traumatic encephalopathy in a professional American wrestler. J Forensic Nurs 6:130-136, 2010

25. Omalu BI, Hamilton RL, Kamboh MI, DeKosky ST, Bailes J: Chronic traumatic encephalopathy (CTE) in a National Football League player: case report and emerging medicolegal practice questions. J Forensic Nurs 6:40-46, 2010

26. Robbins S: Doctors study link between combat and brain disease. Stars and Stripes. January 23, 2010 (http://www. stripes.com/news/doctors-study-link-between-combat-andbrain-disease-1.98394) [Accessed September 23, 2011]

27. Ropper A: Brain injuries from blasts. N Engl J Med 364: 2156-2157, 2011

28. Rosenfeld JV, Ford NL: Bomb blast, mild traumatic brain injury and psychiatric morbidity: a review. Injury 41:437-443, 2010

29. Samatovicz RA: Genetics and brain injury: apolipoprotein E. J Head Trauma Rehabil 15:869-874, 2000

30. Shalev AY: What is posttraumatic stress disorder? J Clin Psychiatry 62 (Suppl 17):4-10, 2001

31. World Health Organization: The ICD-10 Classification of Mental and Behavioural Disorders: Clinical Descriptions and Diagnostic Guidelines. Geneva: World Health Organization, 1992

32. Yule W: Posttraumatic stress disorder in the general population and in children. J Clin Psychiatry 62 (Suppl 17):23-28, 2001

Manuscript submitted July 13, 2011.

Accepted September 14, 2011.

Address correspondence to: Bennet Omalu, M.D., 1132 Junewood Court, Lodi, California 95242. email: bennetomalu@comcast. net. 